\title{
Quantum-Dot Semiconductor Optical Amplifiers: State Space Model versus Rate Equation Model
}

\author{
Hussein Taleb, ${ }^{1}$ Kambiz Abedi, ${ }^{1}$ and Saeed Golmohammadi ${ }^{2}$ \\ ${ }^{1}$ Department of Electrical Engineering, Faculty of Electrical and Computer Engineering, Shahid Beheshti University, \\ G. C., Evin, Tehran 1983963113, Iran \\ ${ }^{2}$ Nanophotonics Group, School of Engineering-Emerging Technologies, University of Tabriz, Tabriz 5166614761, Iran \\ Correspondence should be addressed to Kambiz Abedi; k_abedi@sbu.ac.ir
}

Received 29 November 2012; Accepted 13 February 2013

Academic Editor: Michele Norgia

Copyright (C) 2013 Hussein Taleb et al. This is an open access article distributed under the Creative Commons Attribution License, which permits unrestricted use, distribution, and reproduction in any medium, provided the original work is properly cited.

A simple and accurate dynamic model for QD-SOAs is proposed. The proposed model is based on the state space theory, where by eliminating the distance dependence of the rate equation model of the QD-SOA; we derive a state space model for the device. A comparison is made between the rate equation model and the state space model under both steady state and transient regimes. Simulation results demonstrate that the derived state space model not only is much simpler and faster than the rate equation model, but also it is as accurate as the rate equation model.

\section{Introduction}

During the last decade, the potential capabilities of QDSOAs for use in all-optical signal processing and optical communication systems have been intensively studied. Among these capabilities, ultrafast gain recovery [1-5], high saturated output power [6,7], pattern-effect free signal amplification at high speeds up to $80 \mathrm{~Gb} / \mathrm{s}$ [8-10], pattern-effect free XGMbased wavelength conversion at $160 \mathrm{~Gb} / \mathrm{s}$ [11], capability of operation at $\mathrm{Tb} / \mathrm{s}$ speeds in presence of a control signal [12], amplification of high bit rate multichannel signals [13, 14], low noise figure [15], small dimensions, and integration with other optoelectronic devices such as laser diodes and optical modulators have great importance in any optoelectronic system.

In recent years, several models have been proposed for describing the electrical and optical characteristics of QDSOAs. Among these theoretical models, the most accurate models are based on semiconductor Maxwell-Bloch equations [16-20]. However, the numerical calculations associated with this model are extremely time-consuming and require huge amount of memory. A simplified approach to model QD-SOAs which is known as rate equation model (REM), has demonstrated an excellent agreement with experimental results $[4,21]$. The REM includes a set of coupled differential equations to give details of carrier dynamics and optical properties of QD-SOA. To include the carrier dynamics in the REM, in some papers the electron-hole pairs are considered as exciton and only the carrier dynamics in the conduction band (CB) is taken into account [22-25]. In some other papers, the holes dynamics is included by using quasi-Fermi level in the valence band (VB) [26]. Also, the dynamics of electron and hole are considered separately in some articles [4, 27-31]. This model is known as "electron-hole model" [30], where the rate equations for electrons and holes are written separately. In this paper, we have employed the last approach to give details of the investigated QD-SOA $[29,31]$.

Although the REM of QD-SOA is much simpler and faster than QD Maxwell-Bloch equations, because of time as well as distance dependence of the rate equations, the long computation time is still a big concern in some applications. Furthermore, since the simulation run time is very sensitive to the distance between two adjacent nodes in the spaced distance-time grid, a huge memory may be required during the simulation execution, especially in condition that a long sequence of optical pulses have to be considered in numerical simulations. Therefore, our objective is to derive a simple and efficient dynamical model for QD-SOA that calculates 
the optical gain and output of the device with an acceptable precision. For this purpose, the average values of occupation probabilities along the QD-SOA cavity are considered as the variables of the system and the distance dependence of the REM is eliminated. In the state space model, the QD-SOA is considered as a black box; that is, it is viewed mainly in terms of its input and output characteristics.

This paper is organized as follows. In Section 2, the physical structure of the investigated QD-SOA as well as the rate equations of the device are presented. In Section 3, we derive a SSM for QD-SOA. In Section 4, the effects of the homogeneous and inhomogeneous broadenings are included in the State Space Model of QD-SOAs. Comparison between the results obtained from SSM with results of the REM is presented in Section 5. Finally, Section 6 gives a summary of our work.

\section{Physical Model and Theory}

The investigated device is an InAs/GaAs QD-SOA that operates around $1.3 \mu \mathrm{m}$. The active region of the device consists of seven stacked layers of self-assembled InAs QDs which are grown on a GaAs substrate (see Figure 1).

The detailed band structure of this QD-SOA is illustrated in Figure 2. The self-assembled QDs have 3 nondegenerate energy levels in the $\mathrm{CB}$ and 8 nondegenerate energy levels in the VB, and are accompanied by two dimensional wetting layer (WL) states [29].

The dynamic behavior of the QD-SOA is determined by photon as well as carrier rate equations.

The rate equations of the investigated QD-SOA can be found in $[29,31]$ and are summarized as follows.

Photon rate equation:

$$
\frac{\partial S}{\partial z}=g_{\mathrm{QD}} S-\alpha S
$$

where

$$
g_{\mathrm{QD}}=\sum_{j=0}^{H} g_{j}\left(f_{j}^{n}+f_{j}^{p}-1\right)
$$

where

$$
g_{j}=g_{j}^{\max } \frac{\hbar \omega_{j}^{\max }}{\hbar \omega} \exp \left(\frac{-\left(\hbar \omega-\hbar \omega_{j}^{\max }\right)^{2}}{2 \sigma_{j}^{2}}\right) .
$$

2.1. Electron States Rate Equations. For the GS:

$$
\frac{\partial f_{0}^{n}}{\partial t}=\left(R_{1,0}^{n c}-R_{0,1}^{n e}\right)-R_{0}^{s p}-R_{0}^{s t}
$$

For the $i$ th $\mathrm{ES}(i=1,2)$ :

$$
\frac{\partial f_{i}^{n}}{\partial t}=\left(R_{i+1, i}^{n c}-R_{i, i+1}^{n e}\right)-\left(R_{i, i-1}^{n c}-R_{i-1, i}^{n e}\right)-R_{i}^{s p}-R_{i}^{s t} .
$$

For the WL state:

$$
\frac{\partial w_{n}}{\partial t}=\frac{I}{q V_{a} N_{\mathrm{WL}}}-\left(R_{w_{n}, 2}^{n c}-R_{2, w_{n}}^{n e}\right)-R_{w_{n}}^{s p}
$$

where

$$
\begin{gathered}
R_{i+1, i}^{n c}=\frac{\left(1-f_{i}^{n}\right) f_{i+1}^{n}}{\tau_{i+1, i}^{n}}\left(a_{i+1, i}^{n}+c_{i+1, i}^{n p} w_{p}+c_{i+1, i}^{n n} w_{n}\right), \\
R_{i, i+1}^{n e}=\frac{f_{i}^{n}\left(1-f_{i+1}^{n}\right)}{\tau_{i, i+1}^{n}}\left(a_{i, i+1}^{n}+c_{i, i+1}^{n p} w_{p}+c_{i, i+1}^{n n} w_{n}\right), \\
R_{i}^{s p}=\frac{f_{i}^{n} f_{i}^{p}}{\tau_{i R}}\left(a_{i i}^{n}+c_{i i}^{p} f_{i}^{p}+c_{i i}^{n} f_{i}^{n}\right), \\
R_{i}^{s t}=\frac{v_{g} g_{i}}{N_{Q}}\left(f_{i}^{p}+f_{i}^{n}-1\right) S .
\end{gathered}
$$

2.2. Hole States Rate Equations. For the GS:

$$
\frac{\partial f_{0}^{p}(t)}{\partial t}=\left(R_{1,0}^{p c}-R_{0,1}^{p e}\right)-R_{0}^{s p}-R_{0}^{s t} .
$$

For the $k$ th $\mathrm{ES}(k=1,2)$ :

$$
\frac{\partial f_{k}^{p}(t)}{\partial t}=\left(R_{k+1, k}^{p c}-R_{k, k+1}^{p e}\right)-\left(R_{k, k-1}^{p c}-R_{k-1, k}^{p e}\right)-R_{k}^{s p}-R_{k}^{s t}
$$

For the $k$ th $\mathrm{ES}(3 \leq k \leq 7)$ :

$$
\frac{\partial f_{k}^{p}}{\partial t}=\left(R_{k+1, k}^{p c}-R_{k, k+1}^{p e}\right)-\left(R_{k, k-1}^{p c}-R_{k-1, k}^{p e}\right) .
$$

For the WL state:

$$
\frac{\partial w_{p}}{\partial t}=\frac{I}{q V_{a} N_{\mathrm{WL}}}-\left(R_{w_{p}, 7}^{n c}-R_{7, w_{p}}^{n e}\right)-R_{w_{p}}^{s p},
$$

where $t$ and $z$ are independent variables while all other variables, that is, occupation probabilities, are dependent variables of the REM. Details of the REM and also simulations parameters of the investigated QD-SOA can be found in [31]. The rate equations can be solved for a given injected current and input photon density. By solving the rate equations, the longitudinal and temporal variations of the photon density and occupation probabilities can be determined.

\section{SSM of QD-SOA}

The REM of the investigated QD-SOA was presented in Section 2. As stated before, our objective is to develop a simple and accurate SSM for QD-SOAs. In order to derive the SSM of the QD-SOA, the dependency of the occupation probabilities on distance must be eliminated. By considering the average values of the occupation probabilities as the REM variables, the carrier density along the cavity gets a uniform distribution and consequently, $z$-independent variables will be appeared in the REM of the QD-SOA. By this assumption and after some manipulations, the SSM of the QD-SOA is derived, where the average values of the occupation probabilities are the state variables of the system.

To find out whether the average values of the occupation probabilities are the appropriate state variables of the SSM, 


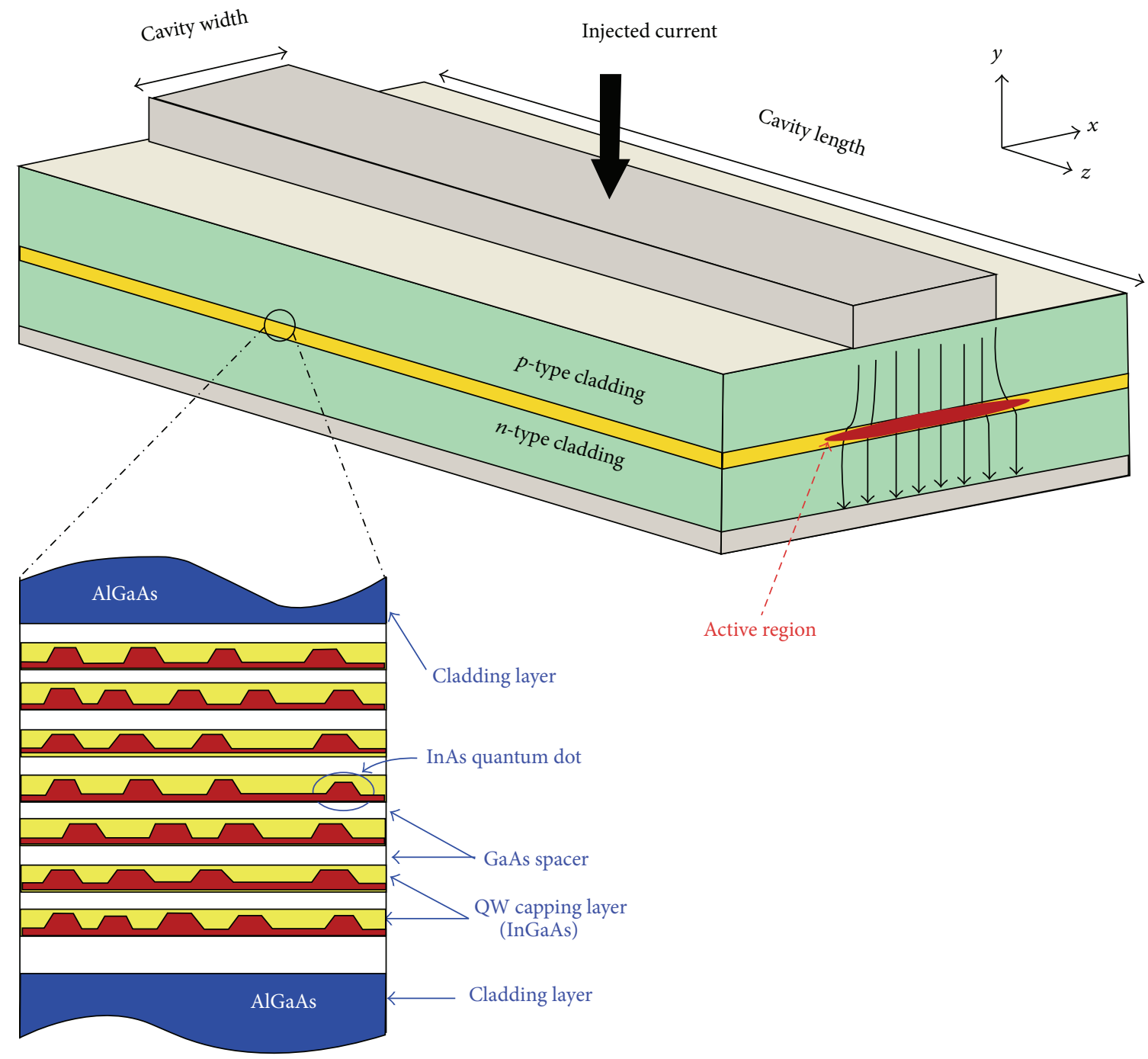

FIGURE 1: Schematic representation of QD-SOA with dots-in-a-well (DWELL) structure, where a sevenfold-stacked self-assembled InAs QD active region is sandwiched between two AlGaAs cladding layers. InAs QDs are covered with a $5 \mathrm{~nm}$ thick InGaAs capping layer. The QD layers are separated by $33 \mathrm{~nm}$ thick GaAs spacer layers which are considered for strain relaxation.

we need to know the effects of uniformity of the occupation probabilities along the cavity on the final results of the REM. Our calculations demonstrate that the variance of the carrier distribution along the cavity does not have a significant impact on the gain dynamics of the amplifier [31]. In other words, the output is weakly dependent on the carrier density variations along the cavity. Consequently, it is reasonable to assume that in the state space model of the QD-SOA, the carrier density distribution along the QD-SOA cavity is completely uniform; that is, we assume that the variance of the carrier density distribution along the cavity is zero. By this assumption, the average values of the occupation probabilities become the state variables of the device [32]. In the following, the details of the mathematical derivation of the SSM are presented.

We denote the distance independent occupation probabilities as $\bar{f}_{k}^{p}(t), \bar{f}_{i}^{n}(t), \bar{w}_{n}$, and $\bar{w}_{p}$. These quantities are the average values of occupation probabilities along the cavity and are considered as the state variables of the QD-SOA in the state space. Therefore, the modal gain of QD-SOA in the SSM takes the following form $\bar{g}_{\mathrm{QD}}(t)$, which we name it as "average modal gain," and is given by

$$
\bar{g}_{\mathrm{QD}}=\sum_{j=0}^{H} g_{j}\left(\bar{f}_{j}^{n}+\bar{f}_{j}^{p}-1\right) .
$$

Since the state variables do not fluctuate along the QD-SOA cavity, the average modal gain will also be unchanged along the cavity. Depending on the supposed model for QDs, the number of state variables is determined. In our model, InAs QDs have totally 11 energy levels. Considering the WL states, the dynamics of our investigated QD-SOA can be described by 13 state variables.

As we know from state space theory, the state update equations of a nonlinear system are generally given by

$$
\dot{x}(t)=F_{1}[x(t), u(t), t],
$$




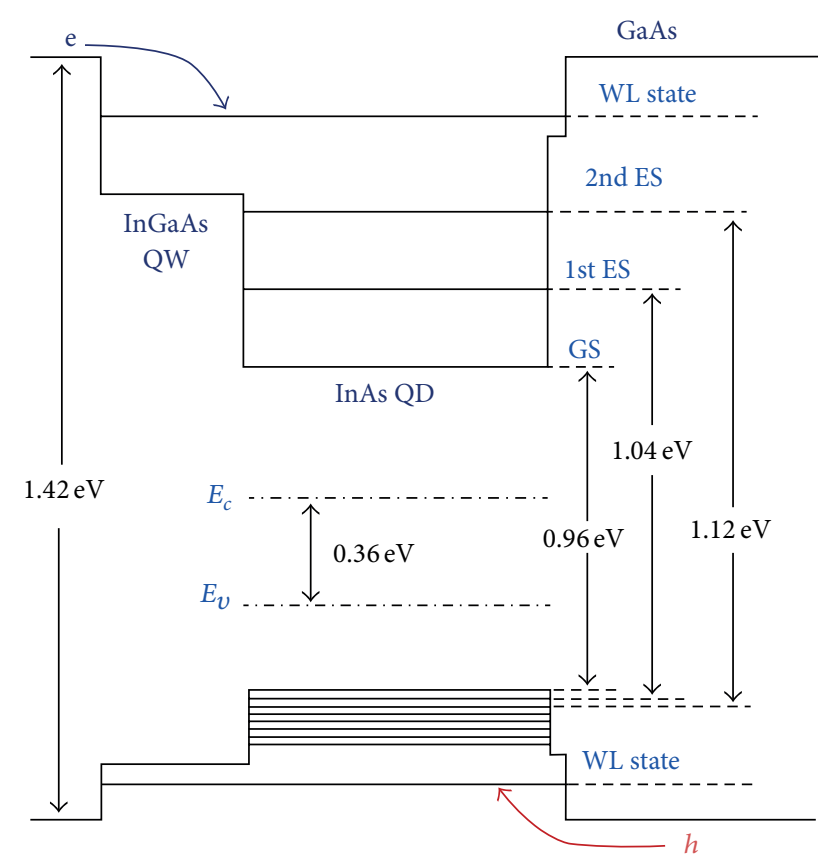

FIGURE 2: The energy band diagram of the active region of the QDSOA. The energy separations of the QD electron and hole states are $70 \mathrm{meV}$ and $10 \mathrm{meV}$, respectively. The radiative transitions are shown in the figure.

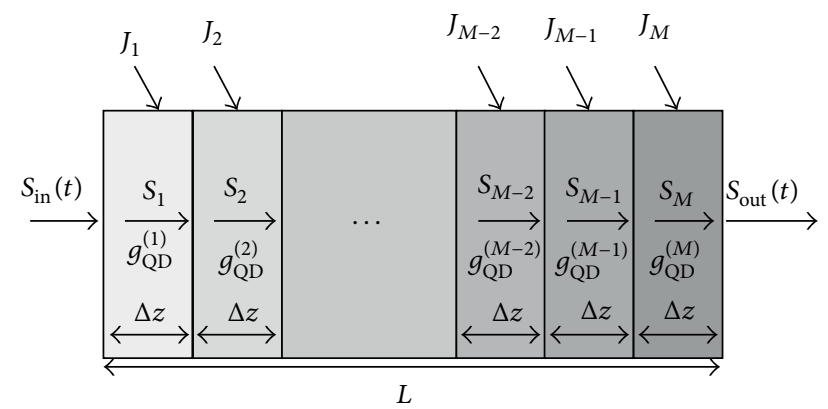

FIGURE 3: Schematic diagram of the QD-SOA cavity which is divided into $\mathrm{M}$ identical sections to be used by FDM, where $L=M \cdot \Delta z$.

where $x(t)$ and $u(t)$ denote, respectively, the state vector and input vector of the system. Also, the output relation of this system is given by

$$
y(t)=F_{2}[x(t), u(t), t]
$$

where $F_{1}$ and $F_{2}$ are nonlinear functions of $t$ as well as $x(t)$ and $u(t)$, and where $y(t)$ denotes the output vector. To derive an SSM for QD-SOA, we begin from photon rate equation and obtain a closed form relation between input and output photon densities. This relation enables us to derive a new relation for stimulated emission rate, and therefore in the next step we rewrite the rate equations of QD-SOA in the form of state update equations. Derivation details are as follows.
The photon rate equation of the QD-SOA can be rewritten using the state variables as follows:

$$
\frac{\partial S}{S}=\left(\bar{g}_{\mathrm{QD}}-\alpha\right) \times \partial z
$$

By integrating (18) we have

$$
\int_{S_{\text {in }}}^{S_{\text {out }}} \frac{\partial S}{S}=\int_{0}^{L}\left(\bar{g}_{\mathrm{QD}}-\alpha\right) \times \partial z .
$$

Therefore, the relation between the input and output photon densities of QD-SOA becomes

$$
S_{\text {out }}(t)=S_{\text {in }}(t) \exp \left(\left(\bar{g}_{\mathrm{QD}}-\alpha\right) \times L\right),
$$

where $S_{\text {in }}$ and $S_{\text {out }}$ are, respectively, the photon densities at the input facet $(z=0)$ and at the output facet $(z=L)$ of the QD-SOA, and $L$ is the cavity length. On the other hand, from (18) we have

$$
S=\frac{1}{\bar{g}_{\mathrm{QD}}-\alpha} \times \frac{\partial S}{\partial z} .
$$

Substituting (21) in (9), one can obtain

$$
R_{i}^{s t}(z, t)=\frac{v_{g} g_{i}\left(\bar{f}_{i}^{n}+\bar{f}_{i}^{p}-1\right)}{N_{\mathrm{Q}}\left(\bar{g}_{\mathrm{QD}}-\alpha\right)} \frac{\partial S(z, t)}{\partial z} .
$$

Integrating (22) from 0 to $L$ and averaging along the cavity, one can get the following expression for stimulated emission rate for the $i$ th energy state as

$$
\bar{R}_{i}^{s t}(t)=\frac{v_{g} g_{i}\left(\bar{f}_{i}^{n}+\bar{f}_{i}^{p}-1\right)}{N_{\mathrm{Q}}\left(\bar{g}_{\mathrm{QD}}-\alpha\right)}\left[\frac{S(L, t)-S(0, t)}{L}\right],
$$

where $S(L, t)=S_{\text {out }}(t), S(0, t)=S_{\text {in }}(t)$. Substituting (20) in (23), we obtain the relation of stimulated emission rate as

$$
\bar{R}_{i}^{s t}(t)=\frac{v_{g} g_{i}\left(\bar{f}_{i}^{n}+\bar{f}_{i}^{p}-1\right) S_{\text {in }}(t)}{N_{\mathrm{Q}}\left(\bar{g}_{\mathrm{QD}}-\alpha\right)}\left[\frac{e^{\left[\bar{g}_{\mathrm{QD}}-\alpha\right] L}-1}{L}\right]
$$

which we name it as "effective" stimulated emission rate. Also, the effective spontaneous emission is given by

$$
\bar{R}_{i}^{s p}(t)=\frac{\bar{f}_{i}^{n} \bar{f}_{i}^{p}}{\tau_{i R}}\left(a_{i i}^{n}+c_{i i}^{p} \bar{f}_{i}^{p}+c_{i i}^{n} \bar{f}_{i}^{n}\right) .
$$

Similarly, the effective electron capture and escape rates are, respectively, given by

$$
\begin{aligned}
& \bar{R}_{i+1, i}^{n c}(t)=\frac{\left(1-\bar{f}_{i}^{n}\right) \bar{f}_{i+1}^{n}}{\tau_{i+1, i}^{n}}\left(a_{i+1, i}^{n}+c_{i+1, i}^{n p} \bar{w}_{p}+c_{i+1, i}^{n n} \bar{w}_{n}\right), \\
& \bar{R}_{i, i+1}^{n e}(t)=\frac{\left(1-\bar{f}_{i+1}^{n}\right) \bar{f}_{i}^{n}}{\tau_{i, i+1}^{n}}\left(a_{i, i+1}^{n}+c_{i, i+1}^{n p} \bar{w}_{p}+c_{i, i+1}^{n n} \bar{w}_{n}\right) .
\end{aligned}
$$




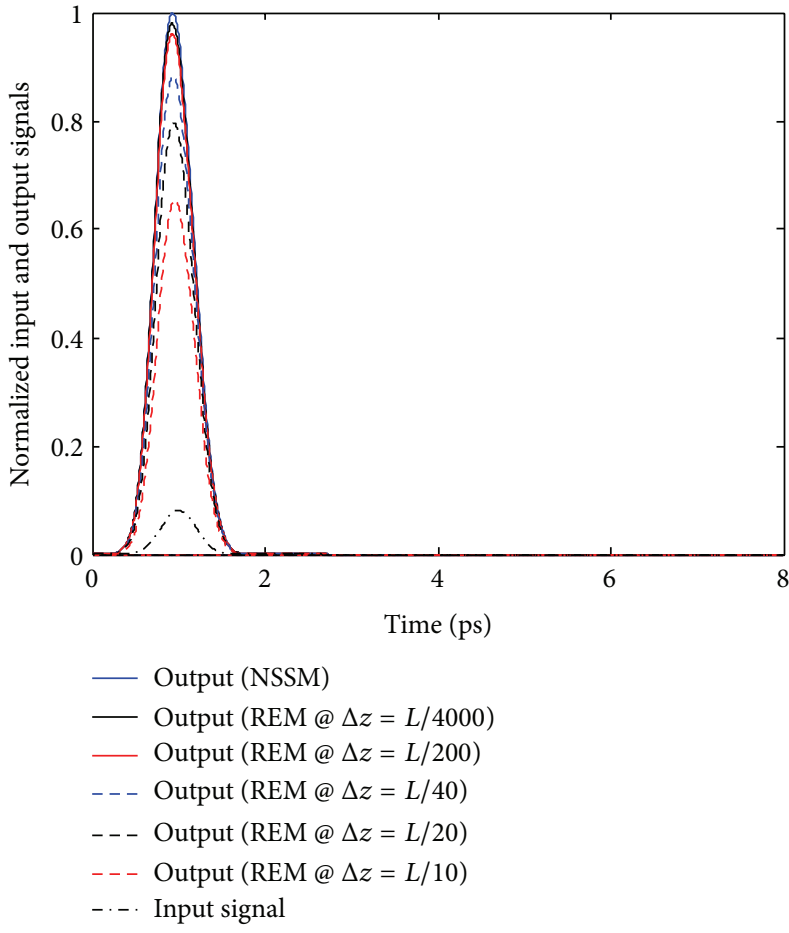

(a)

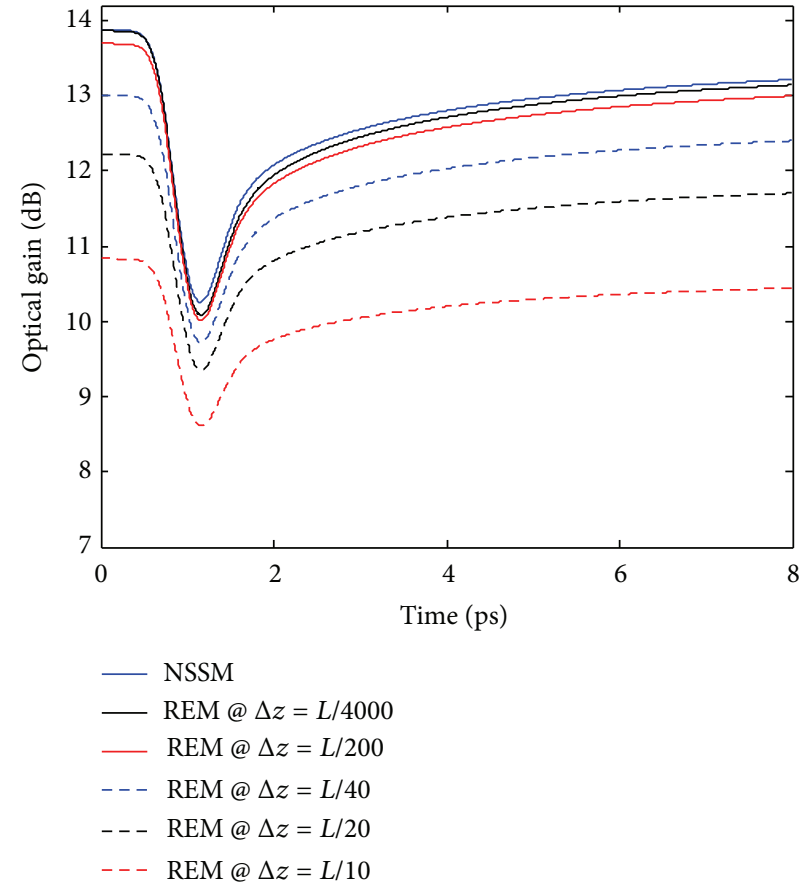

(b)

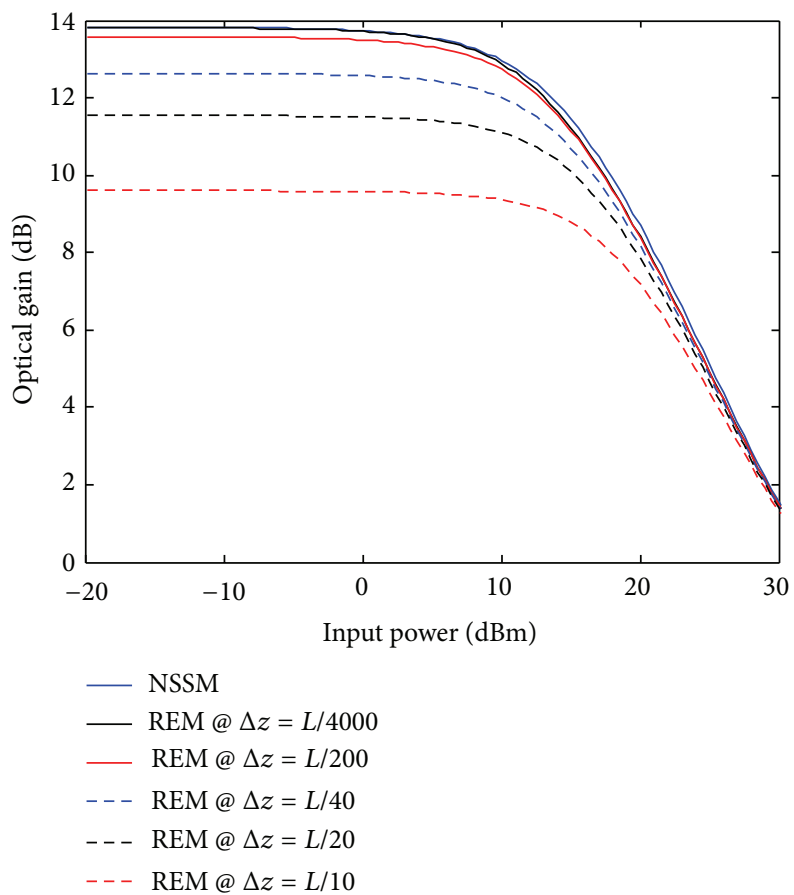

(c)

FIGURE 4: Comparison of the results of the SSM with the results obtained from solving the rate equations of QD-SOA by FDM at $\{\Delta z=$ $L / 4000, \Delta z=L / 200, \Delta z=L / 40, \Delta z=L / 20, \Delta z=L / 10\}$ : (a) gain response, (b) normalized input and output signals, and (c) gain saturation curves@J $=4$.

The effective capture and escape rates for holes in the VB levels are similar to (26) and (27), except that the superscript $n$ is replaced by $p$. Since the rate equations and consequently the state update equations are not explicit functions of time, QD-SOA is an autonomous system. Hence, the SSM of the device takes the following form:

$$
\begin{aligned}
& \dot{x}(t)=F_{1}[x(t), u(t)], \\
& y(t)=F_{2}[x(t), u(t)],
\end{aligned}
$$


where $x=\left[\bar{f}_{0}^{n}, \bar{f}_{1}^{n}, \bar{f}_{2}^{n}, \bar{w}_{n}, \bar{f}_{0}^{p}, \ldots, \bar{f}_{7}^{p}, \bar{w}_{p}\right]^{T}$ is the state vector of the system, $u(t)=S_{\text {in }}(t)$ is the input variable, $y(t)=$ $S_{\text {out }}(t)$ is the output variable, $F_{1}$ is the resultant rate of carriers transition rates, $F_{2}$ describes the relation between the input and output photon densities, that is, (20). Using (24) to (27), we develop the SSM of QD-SOA in the form of (27). The state update equations are given by the following differential equations.

3.1. Electron Levels. For the GS:

$$
\frac{d \bar{f}_{0}^{n}(t)}{d t}=\left(\bar{R}_{1,0}^{n c}-\bar{R}_{0,1}^{n e}\right)-\bar{R}_{0}^{s p}-\bar{R}_{0}^{s t} .
$$

For the $i$ th excited state, where $i=1,2$ :

$$
\frac{d \bar{f}_{i}^{n}(t)}{d t}=\left(\bar{R}_{i+1, i}^{n c}-\bar{R}_{i, i+1}^{n e}\right)-\left(\bar{R}_{i, i-1}^{n c}-\bar{R}_{i-1, i}^{n e}\right)-\bar{R}_{i}^{s p}-\bar{R}_{i}^{s t} .
$$

And for the WL state:

$$
\frac{d \bar{w}_{n}(t)}{d t}=\frac{I}{q V_{a} N_{\mathrm{WL}}}-\left(\bar{R}_{w_{n}, 2}^{n c}-\bar{R}_{2, w_{n}}^{n e}\right)-\bar{R}_{w_{n}}^{s p} .
$$

3.2. Hole Levels. For the GS:

$$
\frac{d \bar{f}_{0}^{p}(t)}{d t}=\left(\bar{R}_{1,0}^{p c}-\bar{R}_{0,1}^{p e}\right)-\bar{R}_{0}^{s p}-\bar{R}_{0}^{s t} .
$$

For the $k$ th excited state, where $k=1,2$ :

$$
\frac{d \bar{f}_{k}^{p}(t)}{d t}=\left(\bar{R}_{k+1, k}^{p c}-\bar{R}_{k, k+1}^{p e}\right)-\left(\bar{R}_{k, k-1}^{p c}-\bar{R}_{k-1, k}^{p e}\right)-\bar{R}_{k}^{s p}-\bar{R}_{k}^{s t} .
$$

For the $k$ th excited state, where $k>2$ :

$$
\frac{d \bar{f}_{k}^{p}(t)}{d t}=\left(\bar{R}_{k+1, k}^{p c}-\bar{R}_{k, k+1}^{p e}\right)-\left(\bar{R}_{k, k-1}^{p c}-\bar{R}_{k-1, k}^{p e}\right) .
$$

And for the WL state:

$$
\frac{d \bar{w}_{p}(t)}{d t}=\frac{I}{q V_{a} N_{\mathrm{WL}}}-\left(\bar{R}_{w_{p}, 7}^{n c}-\bar{R}_{7, w_{p}}^{n e}\right)-\bar{R}_{w_{p}}^{s p} .
$$

The output relation of the SSM is described in (20).

\section{Including the Homogeneous and Inhomogeneous in the State Space Model of QD-SOAs}

In this section, we develop our proposed method for a more comprehensive REM of QD-SOAs. The REM which is used in this paper is proposed by Kim et al. [4]. The investigated device is an InAs/GaAs QD-SOA which operates around $1.3 \mu \mathrm{m}$. Since QDs are grown self-assembly, they have slightly different properties induced by size fluctuations. It is assumed that self-assembled QDs have a size distribution with a Gaussian profile and consist of 181 spectral groups, where QDs in each QD group are assumed identical. The separation between the transition energies of two adjacent groups is considered $\Delta E=1 \mathrm{meV}$. Also, it is assumed that all QDs are spatially isolated and each QD can exchange only with a common carrier reservoir. Details of the physical structure and energy band diagram can be found in $[5,33]$.

The dynamics of each QD group is described by six state variables [33]. Thus, the QD ensemble is described by 1086 state variables $(6 \times 181)$. Including the $\mathrm{QW}$ state in the $\mathrm{CB}$ and VB, the investigated QD-SOA is described by an NSSM with 1088 state variables. The state update equations of the QD-SOA are summarized as follows [33]:

$$
\begin{aligned}
& \frac{d f_{w}^{c(v)}}{d t} \\
& =\frac{J \cdot A}{q N_{W}^{c(v)}}-\frac{\sqrt{f_{w}^{c} f_{w}^{v}}}{\hat{\tau}_{w r}^{c(v)}}+\frac{D_{u}^{c(v)}}{D_{w}^{c(v)}} \\
& \quad \times \sum_{j=1}^{2 M+1} G_{j}^{c(v)}\left(\frac{f_{u, j}^{c(v)}}{\widehat{\tau}_{u w, j}^{c(v)}}\left(1-f_{w}^{c(v)}\right)\right.
\end{aligned}
$$

$$
\left.-\frac{f_{w}^{c(v)}}{\widehat{\tau}_{w u}^{c(v)}}\left(1-f_{u, j}^{c(v)}\right)\right),
$$

$$
\begin{aligned}
\frac{d f_{u, j}^{c(v)}}{d t}= & \frac{f_{w}^{c(v)}}{\widehat{\tau}_{w u}^{c(v)}}\left(1-f_{u, j}^{c(v)}\right)-\frac{f_{u, j}^{c(v)}}{\widehat{\tau}_{u w, j}^{c(v)}}\left(1-f_{w}^{c(v)}\right) \\
& +\frac{D_{e}^{c(v)}}{D_{u}^{c(v)}}\left(\frac{f_{e, j}^{c(v)}}{\widehat{\tau}_{e u}^{c(v)}}\left(1-f_{u, j}^{c(v)}\right)-\frac{f_{u, j}^{c(v)}}{\widehat{\tau}_{u e}^{c(v)}}\left(1-f_{e, j}^{c(v)}\right)\right) \\
& +\frac{D_{g}^{c(v)}}{D_{u}^{c(v)}}\left(\frac{f_{g, j}^{c(v)}}{\widehat{\tau}_{g u}^{c(v)}}\left(1-f_{u, j}^{c(v)}\right)-\frac{f_{u, j}^{c(v)}}{\widehat{\tau}_{u g}^{c(v)}}\left(1-f_{g, j}^{c(v)}\right)\right) \\
& -\frac{\sqrt{f_{u, j}^{c} f_{u, j}^{v}}}{\widehat{\tau}_{u r}^{c(v)}},
\end{aligned}
$$

$$
\begin{aligned}
\frac{d f_{e, j}^{c(v)}}{d t}= & \frac{f_{u, j}^{c(v)}}{\widehat{\tau}_{u e}^{c(v)}}\left(1-f_{e, j}^{c(v)}\right)-\frac{f_{e, j}^{c(v)}}{\widehat{\tau}_{e u}^{c(v)}}\left(1-f_{u, j}^{c(v)}\right) \\
& +\frac{D_{g}^{c(v)}}{D_{e}^{c(v)}}\left(\frac{f_{g, j}^{c(v)}}{\widehat{\tau}_{g e}^{c(v)}}\left(1-f_{e, j}^{c(v)}\right)-\frac{f_{e, j}^{c(v)}}{\widehat{\tau}_{e g}^{c(v)}}\left(1-f_{g, j}^{c(v)}\right)\right) \\
& -\frac{\sqrt{f_{e, j}^{c} f_{e, j}^{v}}}{\widehat{\tau}_{d r}}-\frac{\Gamma}{N_{E, j}^{c(v)}} \\
& \times \sum_{k} \frac{P_{k, \text { in }} g_{j k}^{e}\left[e^{\left(\left[\Gamma g\left(t, \omega_{k}\right)-\alpha_{i}\right] L_{c a}\right)}-1\right]}{E_{k}\left[\Gamma g\left(t, \omega_{k}\right)-\alpha_{i}\right]}\left(f_{e, j}^{c}+f_{e, j}^{v}-1\right),
\end{aligned}
$$




$$
\begin{aligned}
\frac{d f_{g, j}^{c(v)}}{d t}= & \frac{f_{u, j}^{c(v)}}{\widehat{\tau}_{u g}^{c(v)}}\left(1-f_{g, j}^{c(v)}\right)-\frac{f_{g, j}^{c(v)}}{\widehat{\tau}_{g u}^{c(v)}}\left(1-f_{u, j}^{c(v)}\right) \\
& +\frac{f_{e, j}^{c(v)}}{\widehat{\tau}_{e g}^{c(v)}}\left(1-f_{g, j}^{c(v)}\right)-\frac{f_{g, j}^{c(v)}}{\widehat{\tau}_{g e}^{c}}\left(1-f_{e, j}^{c(v)}\right) \\
& -\frac{\sqrt{f_{g, j}^{c} f_{g, j}^{v}}}{\widehat{\tau}_{d r}}-\frac{\Gamma}{N_{G, j}^{c(v)}} \\
& \times \sum_{k} \frac{P_{k, \text { in }} g_{j k}^{g}\left[e^{\left(\left[\Gamma g\left(t, \omega_{\mathrm{k}}\right)-\alpha_{i}\right] L_{c a}\right)}-1\right]}{E_{k}\left[\Gamma g\left(t, \omega_{\mathrm{k}}\right)-\alpha_{i}\right]}\left(f_{g, j}^{c}+f_{g, j}^{v}-1\right) .
\end{aligned}
$$

In (37)-(40), $f_{g, j}^{c(v)}, f_{e, j}^{c(v)}, f_{u, j}^{c(v)}$, and $f_{w}^{c(v)}$ are the state variables of the system. For instance, $f_{g, j}^{c(v)}$ signifies the state variable associated to the ground state of the $j$ th QD group in the conduction (valence) band. Details of this SSM as well as simulation results related to this model can be found in [33].

\section{Simulation Results}

To investigate the accuracy of the SSM, in this section we compare the simulation results of the SSM with those of the REM. We use the finite difference method (FDM) to solve the REM and SSM [31]. To solve the REM using FDM, the cavity length is partitioned into $M$ longitudinal sections (see Figure 3), where the physical quantities in each section are assumed to be constant along the section [31]. To simulate the response of the QD-SOA using the REM and SSM, we need to know the initial state of the system. The initial state (occupation probabilities at $t=0$ ) can be found by solving the REM and SSM at steady state condition for $S_{\text {in }}=0$. Since at steady state the time derivatives are zero, the initial state of the system is equivalent to finding the roots of a system of 13 coupled nonlinear algebraic equations, which can be done by Newton method.

In order to compare the dynamic response of the SSM with that of the REM, the time response of both models are evaluated in condition that a Gaussian shaped pulse with a width of $0.4 \mathrm{ps}$ and $3 \mathrm{pJ}$ energy is applied at the input facet (see Figures 4(a) and 4(b)).

Since the accuracy of the results of the REM depends on the discretization errors, the rate equations of the QD-SOA are solved for different values of $\Delta z$. As seen from the figure, with enhancement the accuracy of numerical calculations, that is, considering smaller values for $\Delta z$, the results of the REM become closer to the results obtained by the SSM. Also, Figure 4(c) illustrates the gain saturation curve of the QDSOA under different values of $\Delta z$. Figure $4(\mathrm{c})$ demonstrates that the SSM of the QD-SOA not only is accurate in the linear region (low input power), but also is accurate at the nonlinear region (under the gain saturation). For $\Delta z=L / 4000$, the results of the REM are very close to the results obtained from the SSM, where the percentage error in calculating the optical gain and output signal is less than $0.5 \%$. These results imply that with lowering the discretization errors, these two models represent even closer results. As is evident, by dividing the cavity length into smaller longitudinal sections the accuracy of the FDM calculations increases. Since the simulation run time of the REMs is very sensitive to the distance steps in the distance-time grid, considering smaller values for $\Delta z$ increases the computation time. The required time for solving the REM is much longer than the simulation run time of the SSM. Furthermore, while the stability of the numerical solving of the state update equations is guaranteed by choosing a small value for $\Delta t$, stable solving of the rate equations is dependent on appropriate selection of both $\Delta t$ and $\Delta z$. Therefore, the convergence problems associated with solving the SSM are reduced compared to REMs.

\section{Conclusion}

A state space model for a typical QD-SOA was proposed. For this purpose, the theoretical basis of the SSM of the QDSOA was developed, where it was shown that the gain of the QD-SOAs is a weak function of distribution of carriers along the cavity. Based on this result, the average values of the occupation probabilities along the QD-SOA cavity were considered as the state variables of the system. Consequently, we derived an SSM for the QD-SOA. We carried out a comparison between the results of the derived SSM and the rate equation model, where both the static and dynamic responses of the QD-SOA were compared with results obtained from the REM. Simulation results showed that the SSM of the QDSOA is accurate in the linear as well as nonlinear region. Numerical calculations demonstrated that not only the SSM is simpler and faster than the rate equations model, but also the convergence problems associated with solving QD-SOA rate equations are decreased.

\section{References}

[1] S. Schneider, U. Woggon, P. Boni et al., "Ultrafast gain recovery dynamics of the excited state in InGaAs quantum dot amplifiers," in Proceedings of the Conference on Lasers and ElectroOptics (CLEO '05), vol. 3, pp. 1674-1676, May 2005, OSA Technical Digest (CD) paper CThH6.

[2] Z. Bakonyi, H. Su, G. Onishchukov et al., "High-gain quantumdot semiconductor optical amplifier for $1300 \mathrm{~nm}$," IEEE Journal of Quantum Electronics, vol. 39, no. 11, pp. 1409-1414, 2003.

[3] S. Schneider, P. Borri, W. Langbein et al., "Excited-state gain dynamics in InGaAs quantum-dot amplifiers," IEEE Photonics Technology Letters, vol. 17, no. 10, pp. 2014-2016, 2005.

[4] J. Kim, M. Laemmlin, C. Meuer, D. Bimberg, and G. Eisenstein, "Theoretical and experimental study of high-speed small signal cross-gain modulation of quantum-dot semiconductor optical amplifiers," IEEE Journal on Selected Topics in Quantum Electronics, vol. 45, pp. 240-248, 2009.

[5] Y. Yi, H. Lirong, X. Meng, T. Peng, and H. Dexiu, "Enhancement of gain recovery rate and cross-gain modulation bandwidth using a two-electrode quantum-dot semiconductor optical amplifier," Journal of the Optical Society of America B, vol. 27, no. 11, pp. 2211-2217, 2010.

[6] T. W. Berg, S. Bischoff, I. Magnusdottir, and J. Mørk, "Ultrafast gain recovery and modulation limitations in self-assembled 
quantum-dot devices," IEEE Photonics Technology Letters, vol. 13, no. 6, pp. 541-543, 2001.

[7] C. Meuer, J. Kim, M. Laemmlin et al., "Static gain saturation in quantum dot semiconductor optical amplifiers," Optics Express, vol. 16, no. 11, pp. 8269-8279, 2008.

[8] T. Vallaitis, C. Koos, R. Bonk et al., "Slow and fast dynamics of gain and phase in a quantum dot semiconductor optical amplifier," Optics Express, vol. 16, no. 1, pp. 170-178, 2008.

[9] M. Sugawara, N. Hatori, M. Ishida et al., "Recent progress in self-assembled quantum-dot optical devices for optical telecommunication: temperature-insensitive $10 \mathrm{~Gb} \mathrm{~s}-1$ directly modulated lasers and $40 \mathrm{~Gb}$ s-1 signal-regenerative amplifiers," Journal of Physics D, vol. 38, no. 13, pp. 2126-2134, 2005.

[10] C. Schmidt-Langhorst, C. Meuer, R. Ludwig et al., "Quantumdot semiconductor optical booster amplifier with ultrafast gain recovery for pattern-effect free amplification of $80 \mathrm{~Gb} / \mathrm{s}$ RZ-OOK data signals," in Proceedings of the 35th European Conference on Optical Communication (ECOC '09), Vienna, Austria, September 2009, Paper 6.2.1.

[11] G. Contestabile, A. Maruta, S. Sekiguchi, K. Morito, M. Sugawara, and K. Kitayama, "160 Gb/s cross gain modulation in quantum dot SOA at1550 nm," in Proceedings of the 35th European Conference on Optical Communication (ECOC '09), pp. 1-2, Vienna, Austria,, 2009, Paper PDP 1.4.

[12] A. Rostami, H. B. A. Nejad, R. M. Qartavol, and H. R. Saghai, " $\mathrm{Tb} / \mathrm{s}$ optical logic gates based on quantum-dot semiconductor optical amplifiers," IEEE Journal of Quantum Electronics, vol. 46, no. 3, pp. 354-360, 2010.

[13] M. Sugawara, N. Hatori, T. Akiyama, Y. Nakata, and H. Ishikawa, "Quantum-dot semiconductor optical amplifiers for high bit-rate signal processing over 40 Gbit/s," Japanese Journal of Applied Physics, vol. 40, no. 5B, pp. L488-L491, 2001.

[14] M. Sugawara, T. Akiyama, N. Hatori, Y. Nakata, H. Ebe, and H. Ishikawa, "Quantum-dot semiconductor optical amplifiers for high-bit-rate signal processing up to $160 \mathrm{~Gb} \mathrm{~s}-1$ and a new scheme of 3R regenerators," Measurement Science and Technology, vol. 13, no. 11, pp. 1683-1691, 2002.

[15] J. L. Xiao and Y. Z. Huang, "Numerical analysis of gain saturation, noise figure, and carrier distribution for quantum-dot semiconductor-optical amplifiers," IEEE Journal of Quantum Electronics, vol. 44, no. 5, pp. 448-455, 2008.

[16] W. W. Chow and S. W. Koch, Semiconductor-Laser Fundamentals: Physics of the Gain Materials, Springer, New York, NY, USA, 1999.

[17] M. van der Poel, E. Gehrig, O. Hess, D. Birkedal, and J. M. Hvam, "Ultrafast gain dynamics in quantum-dot amplifiers: theoretical analysis and experimental investigations," IEEE Journal of Quantum Electronics, vol. 41, no. 9, pp. 1115-1123, 2005.

[18] E. Gehrig and O. Hess, "Mesoscopic spatiotemporal theory for quantum-dot lasers," Physical Review A, vol. 65, no. 3, Article ID 033804, 16 pages, 2002.

[19] W. W. Chow and S. W. Koch, "Theory of semiconductor quantum-dot laser dynamics," IEEE Journal of Quantum Electronics, vol. 41, no. 4, pp. 495-505, 2005.

[20] D. W. Reschner, E. Gehrig, and O. Hess, "Pulse amplification and spatio-spectral hole-burning in inhomogeneously broadened quantum-dot semiconductor optical amplifiers," IEEE Journal of Quantum Electronics, vol. 45, no. 1, pp. 21-33, 2009.

[21] C. Meuer, J. Kim, M. Laemmlin et al., "High-speed smallsignal cross-gain modulation in quantum-dot semiconductor optical amplifiers at $1.3 \mu \mathrm{m}$," IEEE Journal on Selected Topics in Quantum Electronics, vol. 15, no. 3, pp. 749-756, 2009.

[22] M. Sugawara, H. Ebe, N. Hatori et al., "Theory of optical signal amplification and processing by quantum-dot semiconductor optical amplifiers," Physical Review B, vol. 69, no. 23, Article ID 235332, 39 pages, 2004.

[23] A. Bilenca and G. Eisenstein, "On the noise properties of linear and nonlinear quantum-dot semiconductor optical amplifiers: the impact of inhomogeneously broadened gain and fast carrier dynamics," IEEE Journal of Quantum Electronics, vol. 40, no. 6, pp. 690-702, 2004.

[24] O. Qasaimeh, "Characteristics of cross-gain (XG) wavelength conversion in quantum dot semiconductor optical amplifiers," IEEE Photonics Technology Letters, vol. 16, no. 2, pp. 542-544, 2004.

[25] J. Kim and S. L. Chuang, "Small-signal cross-gain modulation of quantum-dot semiconductor optical amplifiers," IEEE Photonics Technology Letters, vol. 18, no. 23, pp. 2538-2540, 2006.

[26] A. V. Uskov, T. W. Berg, and J. Mørk, "Theory of pulsetrain amplification without patterning effects in quantum-dot semiconductor optical amplifiers," IEEE Journal of Quantum Electronics, vol. 40, no. 3, pp. 306-320, 2004.

[27] J. I. Ababneh and O. Qasaimeh, "Simple model for quantumdot semiconductor optical amplifiers using artificial neural networks," IEEE Transactions on Electron Devices, vol. 53, no. 7, pp. 1543-1550, 2006.

[28] O. Qasaimeh, "Effect of doping on the optical characteristics of quantum-dot semiconductor optical amplifiers," Journal of Lightwave Technology, vol. 27, no. 12, pp. 1978-1984, 2009.

[29] O. R. Qasaimeh, "Ultra-fast gain recovery and compression due to auger-assisted relaxation in quantum dot semiconductor optical amplifiers," Journal of Lightwave Technology, vol. 27, no. 13, pp. 2530-2536, 2009.

[30] A. Fiore and A. Markus, "Differential gain and gain compression in quantum-dot lasers," IEEE Journal of Quantum Electronics, vol. 43, no. 4, pp. 287-294, 2007.

[31] H. Taleb, K. Abedi, and S. Golmohammadi, "Operation of quantum-dot semiconductor optical amplifiers under nonuniform current injection," Applied Optics, vol. 50, no. 5, pp. 608617, 2011.

[32] S. B. Kuntze, A. J. Zilkie, L. Pavel, and J. S. Aitchison, "Nonlinear state-space model of semiconductor optical amplifiers with gain compression for system design and analysis," Journal of Lightwave Technology, vol. 26, no. 14, pp. 2274-2281, 2008.

[33] K. Abedi and H. Taleb, "Phase recovery acceleration in quantum-dot semiconductor optical amplifiers," Journal of Lightwave Technology, vol. 30, no. 12, pp. 1924-1930, 2012. 

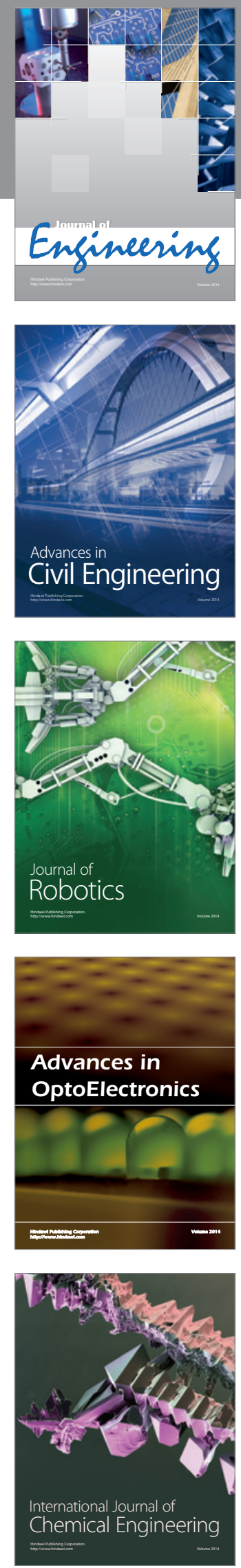

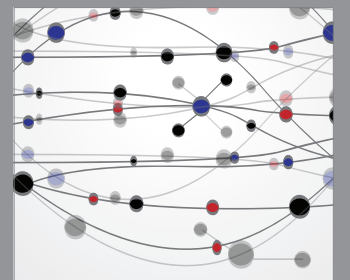

The Scientific World Journal
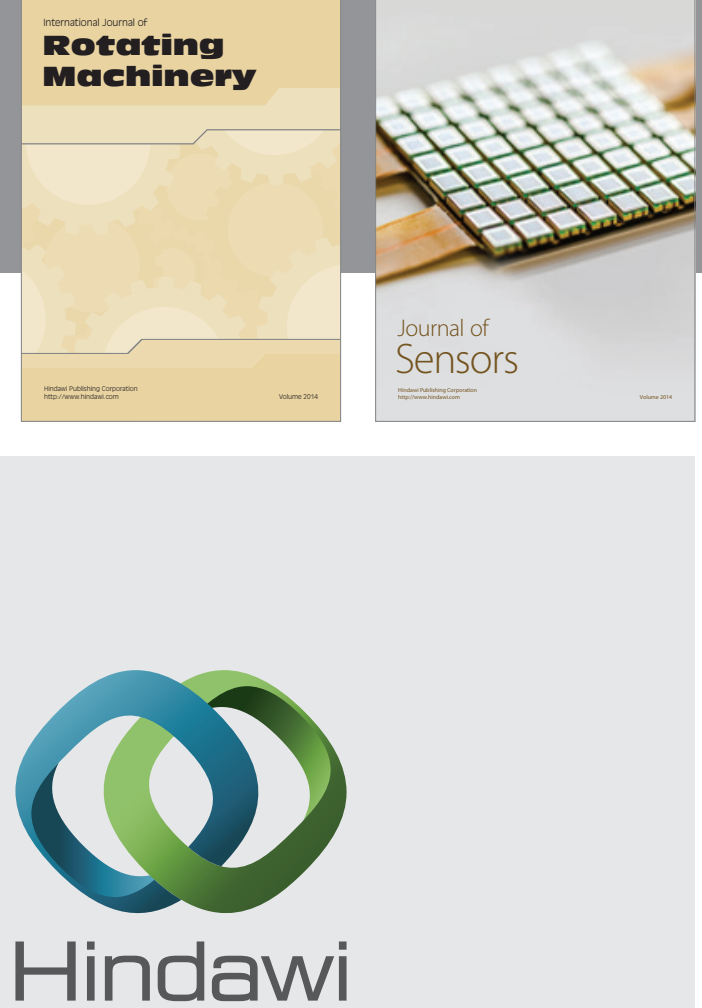

Submit your manuscripts at http://www.hindawi.com
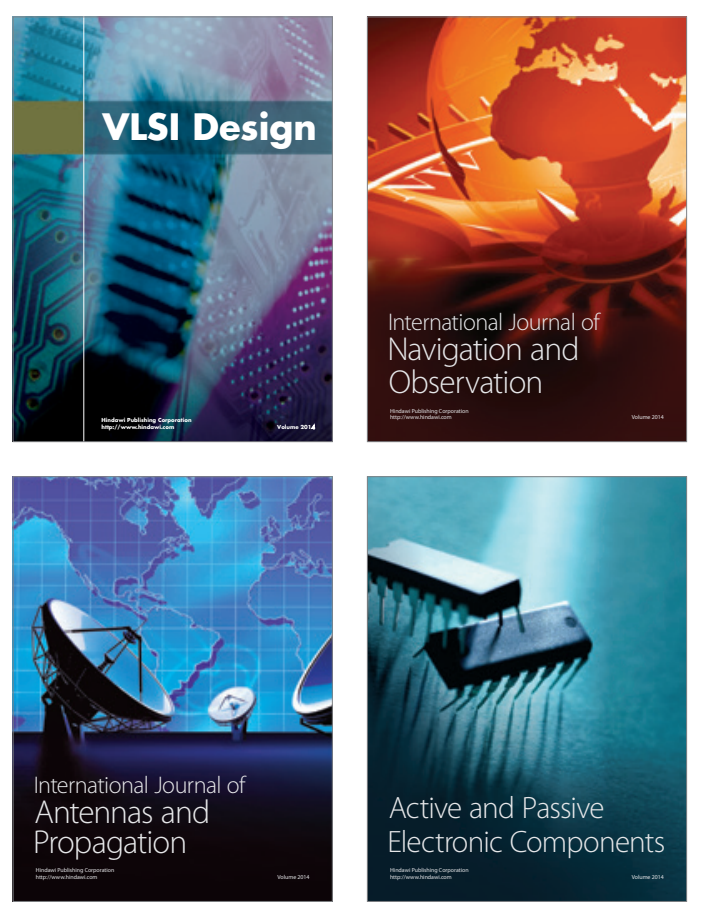
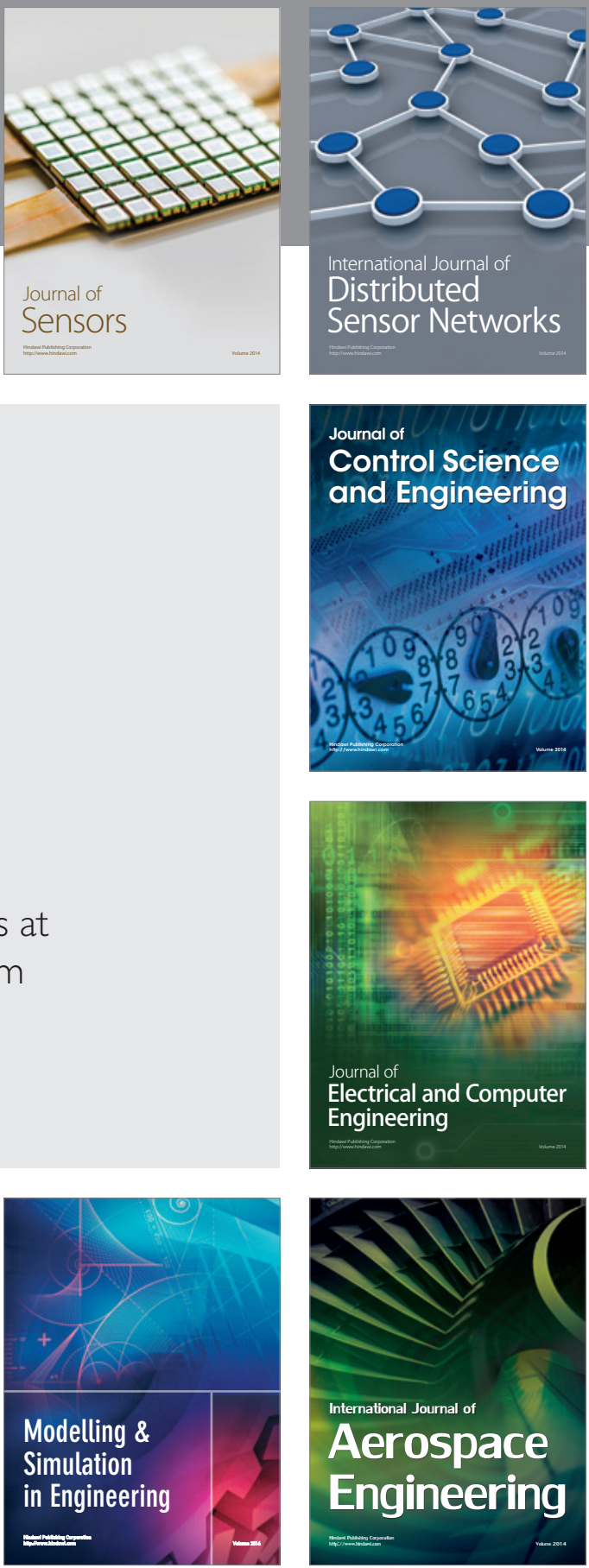

Journal of

Control Science

and Engineering
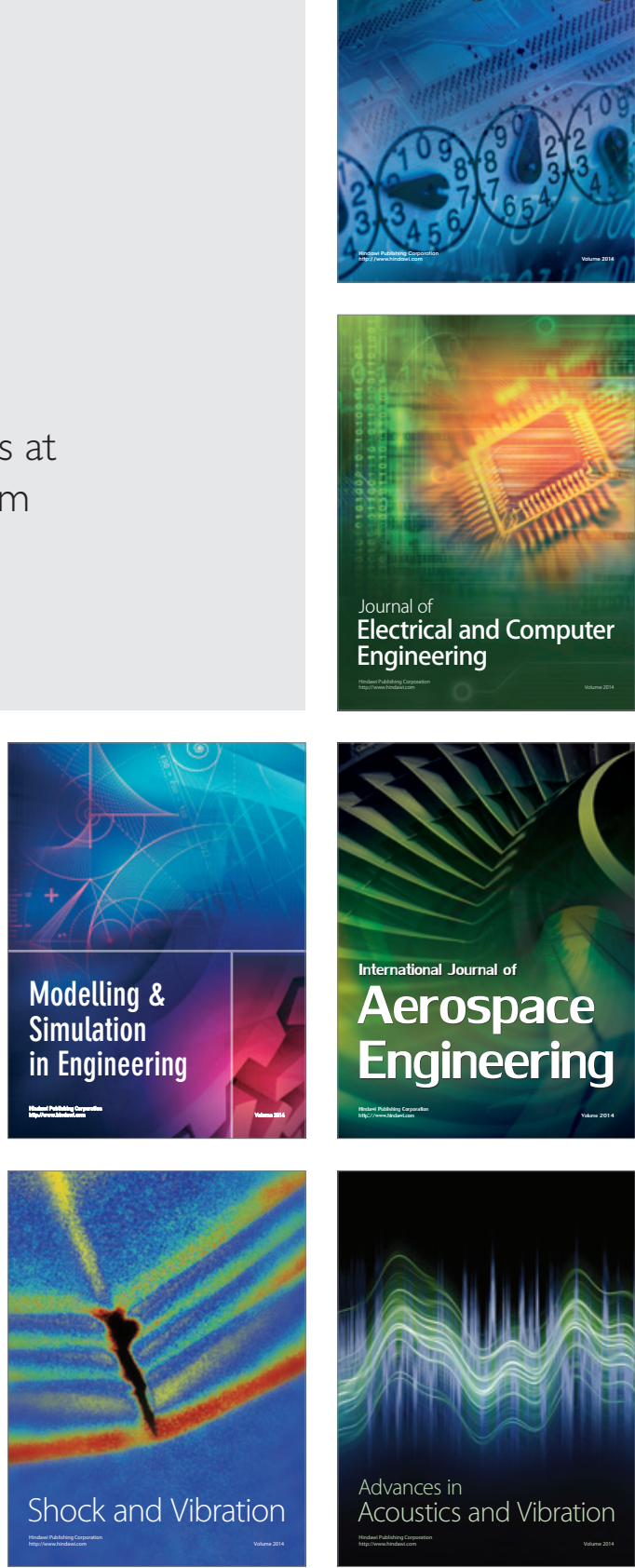